\title{
Microwave-induced off-resonance giant magnetoresistance in ultraclean two-dimensional electron systems
}

\begin{abstract}
Jesus Iñarrea
Escuela Politécnica Superior,Universidad Carlos III, 28911, Leganes, Madrid, Spain.

Received XXXX, revised XXXX, accepted XXXX

Published online XXXX

Key words: Microwaves, radiation coupling, two-dimensional electrons, zero resistance states

* Corresponding author: e-mail jinarrea@fis.uc3m.es, Phone +34 913346249478

We report on theoretical studies of a recently discovered strong radiation-induced magnetoresistance spike obtained in ultraclean two-dimensional electron systems at low temperatures. The most striking feature of this strong spike is that it shows up on the second harmonic of the cyclotron resonance.

We apply the radiation-driven electron orbits model in the ultraclean scenario. Accordingly, we calculate the new average advanced distance by the electron in a scattering event which will define the unexpected resonance spike position. Calculated results are in good agreement with experiments.
\end{abstract}

Copyright line will be provided by the publisher

1 Introduction In the last decade it was discovered that when a high mobility 2DES in a low and perpendicular magnetic field $(B)$ is irradiated, mainly with microwaves (MW), some striking effects are revealed: radiation-induced magnetoresistance $\left(R_{x x}\right)$ oscillations and zero resistance states (ZRS) [1,2]. Different theories and experiments have been proposed to explain these effects [3, 4, 5, 6, 7, 8, 9, 10, 11] but the physical origin is still being questioned. One of the most challenging experimental results, recently obtained[12, 13, 14, 15] consists in a strong resistance spike which shows up far offresonance, at twice the cyclotron frequency, $w \approx 2 w_{c} 12$, [13], were $w$ is the radiation frequency and $w_{c}$ the cyclotron frequency. Remarkably, the only different feature in these experiments 12 13 is the use of ultraclean samples with mobility $\mu \sim 3 \times 10^{7} \mathrm{~cm}^{2} / V s$ and lower temperatures, $T \sim 0.4 K$. Yet, for the previous, "standard", experiments and samples[1] the mobility is lower, $\mu<10^{7} \mathrm{~cm}^{2} / \mathrm{Vs}$ and $T$ higher, $T \geq 1.0 \mathrm{~K}$. In this letter, we theoretically study this radiation-induced $R_{x x}$ spike applying the theory developed by the authors, the radiation-driven electron orbits model[3,4,16]. We show that, in the ultraclean regime, the averaged measured cur- rent results to be the same as the one obtained in a sample with always full contribution to $R_{x x}$ but as if it were irradiated with MW of half frequency $(w / 2)$. Accordingly, the cyclotron resonance is apparently shifted to a new $B$-position around $w \approx 2 w_{c}$.

2 Theoretical Model and Results The radiationdriven electron orbits model, was developed to explain the $R_{x x}$ response of an irradiated 2DEG at low magnetic field [3, 4, 16]. The corresponding time-dependent Schrödinger equation can be exactly solved. We first obtain an exact expression of the electronic wave vector: $\Psi_{N}(x, t) \propto \phi_{n}\left(x-X-x_{c l}(t), t\right)$, where $\phi_{n}$ is the solution for the Schrödinger equation of the unforced quantum harmonic oscillator and $x_{c l}=\frac{e E_{o}}{m^{*} \sqrt{\left(w_{c}^{2}-w^{2}\right)^{2}+\gamma^{4}}} \cos w t=$ $A \cos w t$ where $E_{0}$ is the MW electric field. $\gamma$ is a damping factor for the electronic interaction with the lattice ions giving rise to emission of acoustic phonons. Thus, the electron orbit centers are not fixed, but they oscillate harmonically at $w$. This radiation - driven behavior will affect dramatically the charged impurity scattering and eventually the conductivity. We apply time dependent 


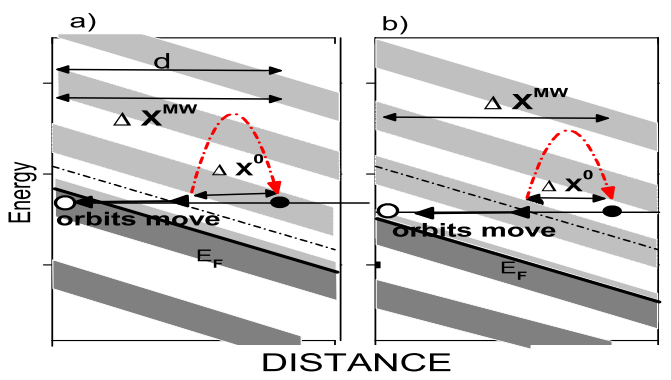

Figure 1 Schematic diagrams presenting the two average electronic scattering scenarios for ultraclean samples. In a), the scattering ends around the central part of a LL (grey stripes). In b) the scattering jump ends in between LL (white stripes). The stripes represent the tilted Landau levels due to static electric field in the $x$ direction. $d$ is the inter-LL distance.

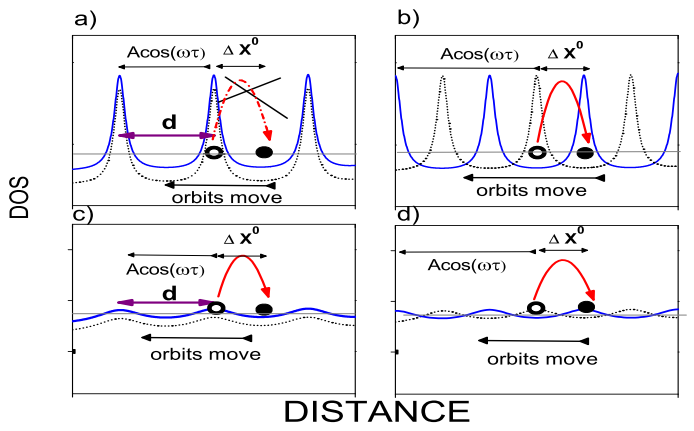

Figure 2 Schematic diagrams of density of Landau states versus energy for ultraclean, a) and b), and standard samples, c) and d). Dotted lines correspond to the density of Landau states before the scattering and single lines after. We present for both kind of samples the two scattering scenarios (see text). $\Delta X^{0}$ is the advanced distance in the dark.

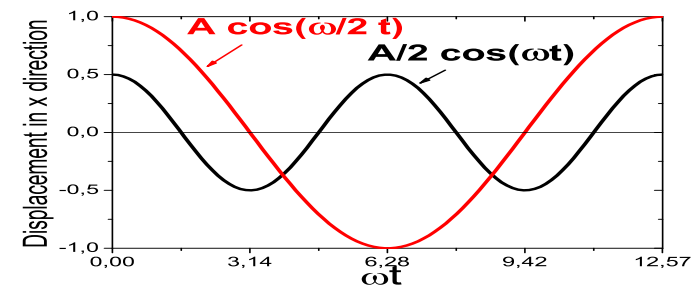

Figure 3 Displacement of two harmonic motions vs time. One with half amplitude $A / 2$ and frequency $w$ and the other with total amplitude $A$ and half frequency $w / 2$. We represent two cycles observing that the net displacement covered by both is the same: $\left|\frac{A}{2} \cos w t\right|=\left|A \cos \frac{w}{2} t\right|$.

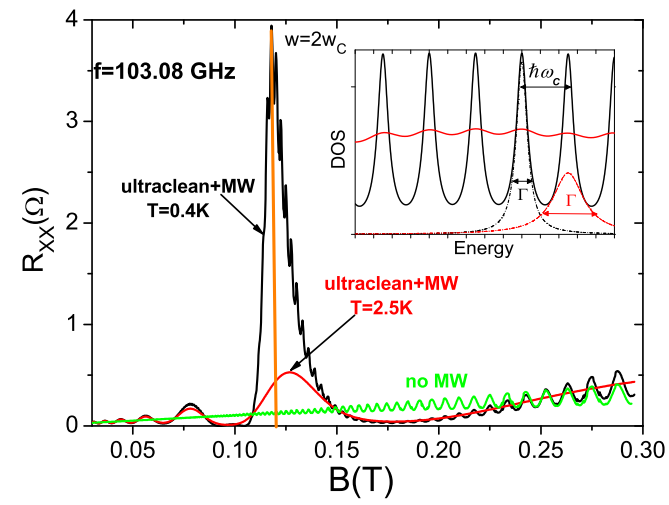

Figure 4 Calculated irradiated magnetoresistance vs static magnetic field for a radiation frequency of $f=$ $103.08 \mathrm{GHz}$, for a ultraclean sample and temperatures, $T=0.4 K$ and $T=2.5 K$. We observe a intense spike at $w \approx 2 w_{c}$. In the inset, density of states for broad and narrow LL.

first order perturbation theory. This allows to calculate the elastic charged impurity scattering rate between two oscillating Landau states, the initial $\Psi_{n}$ and the final state $\Psi_{m}$ [3, 16]: $W_{n, m}=1 / \tau$ being $\tau$ the elastic charged impurity scattering time. Next, we find the average effective distance advanced by the electron in every scattering jump [3, 16], $\Delta X^{M W}=\Delta X^{0}+A \cos w \tau$ where $\Delta X^{0}$ is the advanced distance in the dark. Finally the longitudinal conductivity $\sigma_{x x}$ is given by $\sigma_{x x} \propto \int d E \frac{\Delta X^{M W}}{\tau}$ being $E$ the energy. After some algebra we obtain that the dependence of the magnetoresistance with radiation is mainly given by: $R_{x x} \propto \frac{e E_{o}}{m^{*} \sqrt{\left(w_{c}^{2}-w^{2}\right)^{2}+\gamma^{4}}} \cos w \tau$,

In standard samples (lower mobility) the LL width, $\Gamma$, is large and for experimental magnetic fields[1], it turns out that $\Gamma>\hbar w_{c}$. In this regime due to the strong overlapping of LL, electrons always have an important density of available states where to get to after a scattering event, either around the center of LL or in between them. For ultraclean samples $\Gamma$ is small and generally, $\Gamma<\hbar w_{c}$; the LL hardly overlap each other leaving regions with a low density of states in between (white stripes) This condition will dramatically affect the average advanced distance by electron in every scattering process. Here, we can clearly find two opposite scenarios described in Fig. 1. In this figure, the grey stripes are LL tilted by the action of the DC electric field in the $\mathrm{x}$ direction. We can observe regularly alternating grey (many states) and white (few states) stripes equally spread out. The first scenario corresponds (see Fig. 1a) to an electron being scattered to the central part of a LL where the scattering can be completed; we obtain an important contribution to the conductivity and $R_{x x}$. In Fig. 1b, we describe the second scenario where the electron scatters to a region in between LL with a very low density of states. 
Obviously, in this case there is no much contribution to the average stationary current. In Fig. 2, we present schematic diagrams contrasting the different scattering scenarios for both ultraclean and standard samples. Here, we present the density of Landau states vs distance where dotted lines correspond to the DOS before the scattering and single lines the same but after the scattering. For all presented panels, during the scattering time, Landau states are driven backwards by the action of radiation. In $2 \mathrm{a}$ and $2 \mathrm{~b}$, (ultraclean sample), the scattered electron can either ends in between LL or around the center of one LL respectively. In the first case we hardly obtain current; this happens when $\Delta X_{\text {ultra }}^{M W} \simeq\left(n+\frac{1}{2}\right) d$, where $d$ is the inter-LL distance (see Fig.1a and 2a). In the second case we have full contribution and then the net advanced distance for ultraclean fulfills, $\Delta X_{u l t r a}^{M W} \simeq n d$. In average, the two situations are equally distributed along one cycle of the back and forth MW-driven harmonic orbit motion. In Figs. $2 c$ and $2 d$ we present the same situation for a standard sample. Now, for the two scattering scenarios we always obtain significant contribution to $R_{x x}$. This condition can be mathematically expressed as $\Delta X_{\text {stand }}^{M W} \simeq\left(n+\frac{1}{2}\right) d+n d \simeq 2 n d$, when $n>1$. Comparing both, ultra (contributing part) and standard, we can write $\Delta X_{\text {ultra }}^{M W}=\Delta X_{\text {stand }}^{M W} / 2 \propto \frac{A}{2} \cos w \tau$. Then, regarding MW, the average advanced distance in ultraclean is equivalent to standard but with half amplitude. This point turns out to be crucial, because a harmonic motion with half amplitude $\frac{A}{2}$ and frequency $w$ is in average physically equivalent to another one of amplitude $A$ and half frequency $w / 2$; first, the energy involved in both harmonic motions is the same and, what is more important, averaging in cycles the net displacement performed by both is the same too (see Fig. 3). Thus, we can write: $\left|\frac{A}{2} \cos w t\right|=\left|A \cos \frac{w}{2} t\right|$. Then, adapting this condition to our specific case of MW-driven harmonic motion: $\left|\frac{A}{2} \cos w \tau\right| \simeq\left|A_{2} \cos \frac{w}{2} \tau\right|$ where $A \simeq A_{2}=\frac{e E_{o}}{m^{*} \sqrt{\left(w_{c}^{2}-\left(\frac{w}{2}\right)^{2}\right)^{2}+\gamma^{4}}}$, which is a good approximation according to experimental parameters[12]. The consequence is that the ultraclean harmonic motion behaves as if electrons were driven by radiation of half frequency but with full amplitude (full to contribution to $R_{x x}$ ) during the whole cycle. Therefore, applying next the theory [3] for the ultraclean case it is straightforward to reach an expression for magnetoresistance: $R_{x x} \propto \frac{e E_{o}}{m^{*} \sqrt{\left(w_{c}^{2}-\left(\frac{w}{2}\right)^{2}\right)^{2}+\gamma^{4}}} \cos \frac{w}{2} \tau$ According to it, now the resonance in $R_{x x}$ will take place at $w \approx 2 w_{c}$, as experimentally obtained[12]. The intensity of the $R_{x x}$ spike will depend on the relative value of the frequency term, $\left(w_{c}^{2}-\left(\frac{w}{2}\right)^{2}\right)$, and the damping parameter $\gamma$ in the denominator of the latter $R_{x x}$ expression. When $\gamma$ leads the denominator the spike is smeared out. Yet, in situations where $\gamma$ is smaller than the frequency term, the resonance effect will be more visible and the spike will show up. As we explained above, the parameter $\gamma$ represents the scattering probability of electrons with the lattice ions and the release of the radiation energy in form of acoustic phonons. According to Ando[17] $\gamma$ is linear with the lattice temperature $T: \gamma=\frac{1}{\tau_{a c}} \propto T$. Now by decreasing $T$, it is possible to reach a situation where $\left(w_{c}^{2}-\left(\frac{w}{2}\right)^{2}\right)^{2}>\gamma^{4}$ making visible a resonance effect and, therefore, giving rise to a strong resonance peak at $w \approx 2 w_{c}$. For GaAs and standard experimental parameters[1], we obtain that $\gamma \simeq 7-10 \times 10^{11} s^{-1}$. In a ultraclean experiments [12] it decreases till $\sim 3.5 \times 10^{11}$. This fixes a lower cutoff value for the radiation frequency where the resistance spike could be observed. According to our calculations this would be around $f=w / 2 \pi \approx 40-45 G H z$. In Fig. 4, we present calculated irradiated $R_{x x}$ vs static magnetic field for a radiation frequency of $f=103.08 \mathrm{GHz}$ and a ultraclean sample. The presented results correspond to cases of high and low temperatures. For $T=0.4 K$, we obtain a strong spike at $w \approx 2 w_{c}$ as in experiments[12]. Increasing temperature, $(T \simeq 2.5 K)$ the spike vanishes but the radiation-induced oscillations still remain but with less intensity.

3 Conclusions In this letter we have presented the first theoretical approach to the striking result of the magnetoresistance spike in the second harmonic of the cyclotron frequency. According to our model the strong change in the density of Landau states in ultraclean samples affects dramatically the electron impurity scattering and eventually the conductivity. The final results is that the scattered electrons perceive radiation as of half frequency. Calculated results are in good agreement with experiments.

This work is supported by the MCYT (Spain) under grant MAT2011-24331 and ITN Grant 234970 (EU).

\section{References}

[1] R. G. Mani, J. H. Smet, K. von Klitzing, V. Narayanamurti, W. B. Johnson, and V. Umansky, Nature(London) 420, 646 (2002)

[2] M. A. Zudov, R. R. Du, L. N. Pfeiffer, and K. W. West, Phys.Rev. Lett. 90, 046807 (2003).

[3] J. Iñarrea and G. Platero, Phys. Rev. Lett. 94 016806, (2005); J. Iñarrea and G. Platero, Phys. Rev. B 72193414 (2005)

[4] J. Iñarrea and G. Platero, Appl. Phys. Lett., 89, 052109, (2006); J. Iñarrea, R. Aguado and G. Platero, Europhys Lett. 40, 417, (1997)

[5] A.C. Durst, S. Sachdev, N. Read, S.M. Girvin, Phys. Rev. Lett.91 086803 (2003)

[6] I.A. Dmitriev, M.G. Vavilov, I.L. Aleiner, A.D. Mirlin, D.G. Polyakov, Phys. Rev. B, 71, 115316 , (2005)

[7] X.L. Lei, S.Y. Liu, Phys. Rev. Lett.91, 226805 (2003)

[8] Ryzhii et al, Sov. Phys. Semicond. 20, 1299, (1986)

[9] P.H. Rivera and P.A. Schulz, Phys. Rev. B 70075314 (2004)

[10] R. G. Mani, Appl. Phys. Lett., 92, 102107, (2008); R. G. Mani, C. Gerl, S. Schmult, W. Wegscheider, V. Umansky, Phys. Rev. B 81, 125320 (2010)

[11] Junren Shi and X.C. Xie, Phys. Rev. Lett. 91, 086801 (2003)

[12] Yanhua Dai, R.R. Du, L.N. Pfeiffer and K.W. West, Phys. Rev. Lett., 105, 246802, (2010). 
[13] A.T. Hatke, M.A. Zudov,, L.N. Pfeiffer, K.W. West, Phys. Rev. B, 83, 121301(R), (2011)

[14] Y.H. Dai, K. Stone, I. Knez, C. Zhang, R.R. Du, C.L. Yang, L.N. Pfeiffer, K.W. West, Phys. Rev. B, 84, 241303, (2011)

[15] A.T. Hatke, M.A. Zudov, L.N. Pfeiffer, K.W. West, Phys, Rev, B, 83, 201301, (2011)

[16] J. Inarrea, C. Lopez-Monis, A.H. MacDonald and G. Platero, Appl. Physl Lett., 91, 252112, (2007); J. Inarrea, Appl. Physl Lett. 90, 262101, (2007); J. Iñarrea and G. Platero, Appl. Phys Lett. 93, 062104, (2008);J. Iñarrea, Appl. Phys Lett. 92, 192113,(2008);J. Iñarrea, Phys. Status Solidi, 6, 271, (2012) 192113,(2008)

[17] T. Ando, A. Fowler and F. Stern, Rev. Mod. Phys.,54,(1982). 